\title{
Electromagnetic Design and Loss Calculations of a 1.12-MW High-Speed Permanent-Magnet Motor for Compressor Applications
}

\author{
Fengge Zhang, Guanghui Du, Tianyu Wang, Fengxiang Wang, Wenping Cao, Senior Member, IEEE, James L. Kirtley, Life Fellow,
} IEEE

\begin{abstract}
Electromagnetic design of a 1.12-MW, 18,000-rpm high-speed permanent-magnet motor (HSPMM) is carried out based on the analysis of pole number, stator slot number, rotor outer diameter, air gap length, permanent magnet (PM) material, thickness and pole arc. The no-load and full-load performance of the HSPMM is investigated in this paper by using 2-D finite element method (FEM). In addition, the power losses in the HSPMM including core loss, winding loss, rotor eddy current loss and air friction loss are predicted. Based on the analysis, a prototype motor is manufactured and experimentally tested to verify the machine design.
\end{abstract}

Index Terms - Electromagnetic design, finite element method, high speed, loss calculation, permanent magnet motors.

\section{INTRODUCTION}

$I^{n}$ n recent years, high-speed permanent-magnet motors (HSPMMs) are gaining in popularity in industry due to their high power density, low weight and high efficiency [1]-[3]. They are employed in applications such as compressors, motor tools, gas turbines, flywheels, centrifuges, and vacuum pumps [4]-[6]. In conventional compressor systems, a gearbox is often used, which increases noise and reduces the system efficiency. In the air compressor applications, the HSPMM is directly attached to the motor shaft so that the gearbox is eliminated. However, in industry, there are increasing demands for cutting-edge technologies involving improved materials, rotor strength, electromagnetic design, loss calculations and heat dissipation, rotor dynamics, detection technology and automatic control systems [7]-[9]. High-power

Manuscript received December 1, 2014; revised August 28, 2015; accepted September 28, 2015. Paper TEC-00811-2014.R1 approved for publication in the IEEE TRANSACTIONS ON ENERGY CONVERSION. This work was supported in part by program for Changjiang Scholars and Innovative Research Team in Universitythe of the Ministry of Education of China under Grant IRT1072, the National Natural Science Foundation of China under Grant 51207094, and the FP7 International Research Staff Exchange Scheme under Project 318925.

F. Zhang, G. Du and F. Wang are with the School of Electrical Engineering, Shenyang University of Technology, Shenyang, 110870, China (e-mail: zhangfg@sut.edu.cn; duguanghui1104@163.com; wangfx_2008@ yahoo.com. cn).

T. Wang is with the School of Mechanical Engineering, Shenyang Institute of Engineering, Shenyang, 110136, China (e-mail: lnwangtianyu@aliyun.com).

W. Cao and J. L. Kirtley are with the Department of Electrical Engineering and Computer Science, Massachusetts Institute of Technology, Cambridge, MA, 02139, U.S.A., (e-mail: wencao@mit.edu; kirtley@mit.edu). high-speed motors pose some technical challenges owing to strong thermal and mechanical constraints that limit their maximum power and speed. The maximum power in air compressor applications at the speed range of 20,000-100,000 rpm is presently limited to 1.5 MW [10]. The estimated maximum power of the high-speed PM motors for compressor applications is $1.5 \mathrm{MW}$ at 20,000 rpm based on thermal and mechanical constraints [11][12]. Therefore, the optimized design of HSPMMs for a target industrial application is a comprehensive process which generally involves multi-physical coupled analysis (e.g. electrical, thermal, and mechanical constraints should be simultaneously implemented) [13][14]. As a key part of multiphysics design, a proper electromagnetic design should lead to low overall losses and excellent electromagnetic performance. It should also aid in other designs such as rotor strength and cooling system. However, effective heat dissipation for high-power HSPMMs becomes of critical importance due to their high losses within a compact motor frame [15][16].

In this paper, a 1.12-MW, 18,000-rpm HSPMM is designed for compressor applications. The effects of pole number, stator slot number, rotor outer diameter, air gap length, PM material and magnetization directions on electromagnetic performance of the HSPMM are analyzed. The performance in terms of air-gap flux density, induced emf, full-load torque and full-load current is extensively investigated using 2-D finite-element method (FEM). In addition, the power losses in the HSPMM including core iron loss, stator winding loss, rotor eddy current loss and air friction loss are predicted numerically. Then a prototype HSPMM is manufactured and experimentally tested on at no-load and full-load. An experimental platform is set up and test results have validated numerical models and the effectiveness of the HSPMM design.

\section{DESIGN CONSIDERATIONS OF THE HSPMM}

For the HSPMM, the centrifugal force of the rotor surface at high-speed rotation is expressed as [17]

$$
F=\frac{m v^{2}}{r}=A_{r} \rho D^{2} \omega^{2}
$$


where $\rho$ is the density of rotor material, $A_{\mathrm{r}}$ is the cross-sectional area of the rotor, $D$ is the outer diameter of the rotor, $\omega$ is the angular speed of the rotor.

The centrifugal stress on the rotor surface is given by [17]:

$$
\sigma=\frac{F}{A_{r}}=\rho v^{2}
$$

where $v$ is the line velocity at the rotor outer surface. For a given material, this centrifugal stress should be less than its mechanical strength with a safety margin, as in Eq. 3 [18].

$$
\sigma \leq \frac{[\sigma]}{S}
$$

where $[\sigma]$ is the yield stress of the rotor material, and $S$ is the safety factor.

The maximum line velocity on the rotor surface $\left(v_{\max }\right)$ is given by [18],

$$
v_{\max }=\sqrt{\frac{[\sigma]}{S \rho}}
$$

The maximum rotor diameter $\left(D_{\max }\right)$ can be expressed as

$$
D_{\max }=\frac{2 v_{\max }}{\omega}
$$

The rotor outer diameter $D$ should be less than $D_{\max }$, and the inner diameter of the stator is

$$
D_{\text {a }}=D+\delta
$$

where $\delta$ is the air gap. The stack length $\left(L_{\mathrm{a}}\right)$ can be found by [19],

$$
D_{\mathrm{a}}^{2} L_{\mathrm{a}}=\frac{6.1 \times 10^{4}}{a_{\mathrm{p}} k_{\mathrm{Nm}} K_{\mathrm{dp}} A B_{\delta}} \cdot \frac{p^{\prime}}{n_{N}}
$$

where $p$ ' is the apparent power, $a_{\mathrm{p}}$ is the pole arc coefficient, $K_{\mathrm{Nm}}$ is the air-gap flux factor, $K_{\mathrm{dp}}$ is the stator winding factor, $A$ is the electric loading, $B_{\delta}$ is the air-gap flux density, $n_{\mathrm{N}}$ is the rated speed.

For motor, the apparent power can be expressed as [19]

$$
\begin{gathered}
p^{\prime}=m E_{N} I_{N}=K_{E} \frac{p_{N}}{\eta \cos \varphi} \\
K_{E}=\frac{E_{N}}{U_{N}}
\end{gathered}
$$

where $m$ is the number of phases, $E_{N}$ is the induced emf at full-load, $I_{N}$ is the rated current, $P_{N}$ is the rated power, $\eta$ is the rated efficiency, $\cos \varphi$ is the power factor, $K_{E}$ is the ratio of $E_{N}$ over $U_{N}$. In order to obtain the initial size of the motor, $K_{E}$ can be estimated by the empirical method.

For sizing the HSPMM, it should take account of pole number, stator slot number, rotor outer diameter, axial length, air-gap length, PM material and magnetization orientation.

\section{A. Pole Number}

For high-speed motors, a 2-pole or 4-pole rotor structure is commonly chosen for HSPMMs. The motor with a 2-pole rotor structure can have a better utilization of PMs and lower power losses than a 4-pole counterpart [1]. However, the disadvantage lies in the stator's end-windings. Different designs using 2-pole and 4-pole rotor structures are shown in Table I for comparison. Cases 1 and 2 use a 2-pole structure while case 3 uses a 4-pole structure. As can be seen from this table, the coil half-turn length of cases 1 and 2 are 1303 and $1222 \mathrm{~mm}$, respectively, which are much greater than case 3 . Long end-windings induce extra power losses and also impact on the rotor dynamics. As a result, a 4-pole structure is adopted for the HSPMM.

$$
\text { TABLE I }
$$

DESIGNS WITH DIFFERENT POLES FROM FEM

\begin{tabular}{|l|c|c|c|}
\hline \multicolumn{1}{|c|}{ Design case } & 1 & 2 & 3 \\
\hline Pole number & 2 & 2 & 4 \\
\hline Stack length (mm) & 600 & 490 & 420 \\
\hline Number of conductors per slot & 6 & 8 & 6 \\
\hline Coil half-turn length (mm) & 1303 & 1222 & 870 \\
\hline
\end{tabular}

\section{B. Stator Slot Number}

The stator slot number affects cogging torque and associated power losses. Table II shows the cogging torque and PM eddy current loss in the HSPMM in relation to the number of stator slots. From Table II, the cogging torque is reduced significantly as the stator slot number increases. Especially, the use of 27 slots leads to fractional slot windings which in turn can effectively reduce the cogging torque. Similarly, the PM eddy current loss is reduced significantly with the stator slot number. Therefore, a multi-slot stator structure with 27 slots is adopted for the HSPMM.

TABLE II

COGGING TORQUE AND PM EDDY CURRENT LoSS WITH STATOR SLOT NUMBERS FROM FEM

\begin{tabular}{|c|c|c|c|c|}
\hline Stator slot number & 12 & 24 & 27 & 36 \\
\hline Cogging torque $(\mathrm{Nm})$ & 146 & 31 & 0.5 & 16 \\
\hline PM eddy current loss $(\mathrm{kW})$ & 35 & 2.6 & 1.2 & 0.4 \\
\hline
\end{tabular}

\section{Rotor Outer Diameter and Axial Length}

The rotor dimension is also among important parameters in the machine design. Table III shows the PM eddy current loss and axial length versus different rotor outer diameters. During the numerical study, the output power and no-load emf are kept identical. When the rotor outer diameter is set to $196 \mathrm{~mm}$, the PM eddy current loss is about $4300 \mathrm{~W}$ and the axial length is $430 \mathrm{~mm}$. For the diameter of $166 \mathrm{~mm}$, the PM eddy current loss is about $1250 \mathrm{~W}$ and the axial length is $600 \mathrm{~mm}$. Clearly, a larger rotor outer diameter will result in a greater PM eddy current loss and a greater centrifugal force. Therefore, there is a balance to strike between the rotor outer diameter and the rotor axial length.

TABLE III

AXIAL LENGTH AND PM EDDY CURRENT LOSS WITH ROTOR OUTER DIAMETERS FROM FEM

\begin{tabular}{|l|c|c|c|c|}
\hline Rotor outer diameter $(\mathrm{mm})$ & 166 & 176 & 186 & 196 \\
\hline Axial length $(\mathrm{mm})$ & 600 & 535 & 480 & 430 \\
\hline PM eddy current loss $(\mathrm{W})$ & 1250 & 1860 & 2910 & 4300 \\
\hline
\end{tabular}

\section{Air-Gap Length}

Table IV shows the rotor eddy current loss and cogging torque as a function of the air-gap length. It is found that the cogging torque and PM eddy current loss decrease as the airgap length increases. Since 27 stator slots are used, the reduction in an already small cogging torque is unnecessary. When the air-gap length is $1 \mathrm{~mm}$, the PM eddy current loss is 
$4.2 \mathrm{~kW}$. If it increases to $4 \mathrm{~mm}$, the PM eddy current loss is less than $1 \mathrm{~kW}$. Clearly, a greater air-gap length can significantly mitigate the rotor eddy current loss. In order to meet electromagnetic specifications and rotor safety requirements, the use of PMs and a retaining sleeve is a prerequisite, which adversely impacts on heat dissipation. Therefore, a 3-mm air-gap length is chosen for the HSPMM. TABLE IV

COGGING TORQue AND PM EdDy CURRENT LOSS With AIR-GAP LENGTHS FROM FEM

\begin{tabular}{|l|c|c|c|c|}
\hline Air-gap length $(\mathrm{mm})$ & 1 & 2 & 3 & 4 \\
\hline PM eddy current loss $(\mathrm{W})$ & 4200 & 2320 & 1250 & 750 \\
\hline Cogging torque $(\mathrm{Nm})$ & 2.8 & 1.6 & 1.1 & 0.8 \\
\hline
\end{tabular}

E. PM Materials, thickness and pole arc

In the PM motor design, sintered $\mathrm{NdFeB}$ and $\mathrm{SmCo}$ are usually used for various applications. By comparison, the tensile strength of $\mathrm{NdFeB}(80 \mathrm{MPa})$ is much higher but its maximum withstand temperature $\left(150^{\circ} \mathrm{C}\right)$ is much lower than $\mathrm{SmCo}\left(30 \mathrm{MPa}\right.$ and $350^{\circ} \mathrm{C}$ ). Carbon fiber is commonly used in the retaining sleeve owing to its light weight and high strength (1200MPa). The stress curve of PM with carbon fiber thickness at $150^{\circ} \mathrm{C}$ is shown in Fig. 1. The simulation results are obtained by the 3D FEM based on the 4-pole PM structure. It is seen from Fig. 1, the minimal retaining sleeve thickness for SmCo protection is close to $30 \mathrm{~mm}$, which is about four times of the length required for $\mathrm{NdFeB}$. As a rule of thumb, the maximum stress of PMs and retaining sleeve at rated speed should not exceed 0.8 times the tensile strength of the materials in order to maintain the rotor's integrity. Therefore $\mathrm{NdFeB}$ is selected as the PM material.

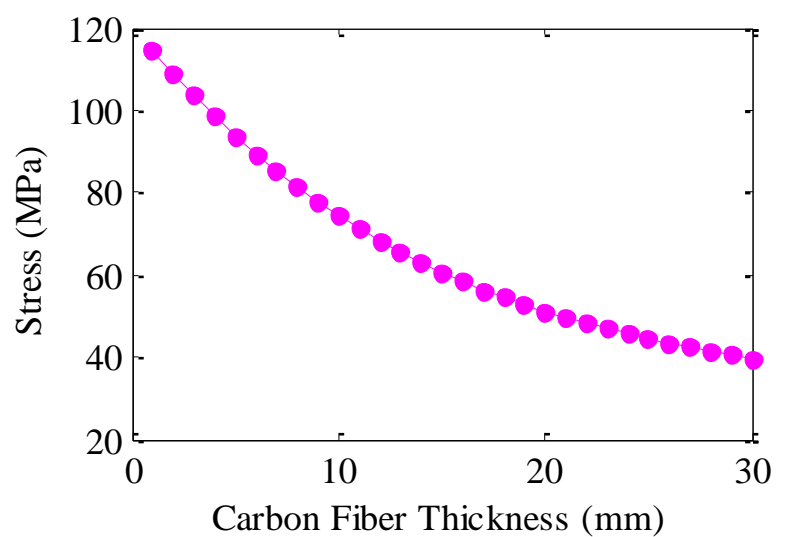

Fig. 1. PM stress with sleeve thickness at $150^{\circ} \mathrm{C}$.

The thickness of PM and pole arc coefficient are important parameters for motor design. Initial values can be estimated by [20]

$$
\begin{gathered}
h_{m}=\frac{2 k_{s} k_{\delta} B_{\delta} \delta}{\mu_{0} H_{c}} \\
A_{m}=\frac{2 \sigma A_{\delta} B_{\delta}}{B_{r}}=\frac{h_{m} r_{m} \alpha_{p} \pi}{p} \\
\alpha_{p}=\frac{2 \sigma A_{\delta} B_{\delta} p}{\pi h_{m} r_{m} B_{r}}
\end{gathered}
$$

where $h_{m}$ is the thickness of PMs, $k_{s}$ is the saturation coefficient of stator iron, $k \delta$ is the saturation coefficient of air-gap, $B \delta$ is the air-gap flux density, $\delta$ is the air-gap length, $\mu_{0}$ is the permeability of air, $H_{c}$ is the coercive force of PMs. $A_{m}$ is the PM area under one pole, $\sigma$ is the leakage coefficient, $A_{\delta}$ is the air-gap area under one pole, $B_{r}$ is the magnetic flux density of PMs, $r_{m}$ is the equivalent radius of PMs, $a_{p}$ is the pole arc coefficient, $P$ is the pole number.

The motor Performance with different PM thickness and pole arc coefficient are tabulated in Tables $\mathrm{V}$ and VI, respectively. It can be seen from Table V that a greater PM thickness increases air-gap flux density and power factor, so as a larger PM stress, which will affect the mechanical integrity of the rotor. Therefore, there is a balance to strike between air-gap flux density and mechanical strength of the rotor.

In Table VI, PM loss, cogging torque and no-load current gradually increase with the pole arc coefficient. That is, more harmonics are generated with larger pole arc coefficient. Nonetheless, a smaller coefficient will lead to a lower magnetic flux and a greater stack length to maintain the same no-load emf.

TABLE V

MOTOR PERFORMANCE WITH PM THICKNESS

\begin{tabular}{|c|c|c|c|c|c|c|}
\hline$h_{\mathrm{m}}(\mathrm{mm})$ & 15 & 16 & 17 & 18 & 19 & 20 \\
\hline$B_{\delta}(\mathrm{T})$ & 0.49 & 0.503 & 0.514 & 0.523 & 0.532 & 0.534 \\
\hline Power Factor & 0.95 & 0.96 & 0.975 & 0.985 & 0.99 & 0.99 \\
\hline PM Stress (MPa) & 60 & 63 & 65 & 69 & 72 & 75 \\
\hline
\end{tabular}

Motor Performance With Pole ARC CoefFicient

\begin{tabular}{|c|c|c|c|c|c|}
\hline Pole Arc Coefficient & 0.7 & 0.75 & 0.8 & 0.9 & 1 \\
\hline PM loss(W) & 1050 & 1120 & 1174 & 1268 & 1340 \\
\hline Cogging torque(Nm) & 0.4 & 0.6 & 0.94 & 2.23 & 3.6 \\
\hline No-load current(A) & 6 & 17 & 31 & 46 & 58 \\
\hline Stack length $(\mathrm{mm})$ & 413 & 400 & 390 & 381 & 374 \\
\hline
\end{tabular}

F. Electromagnetic Analysis of the HSPMM

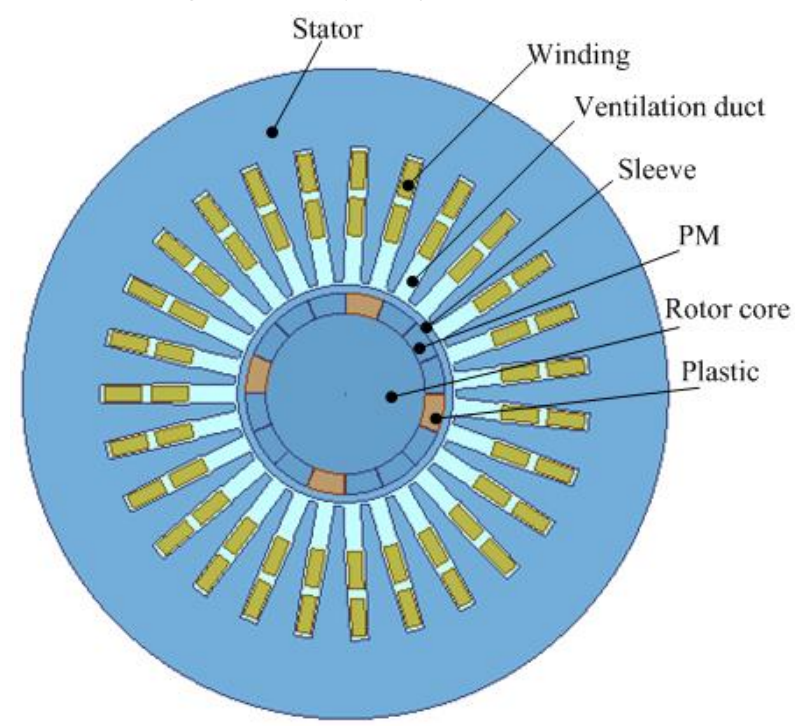

Fig. 2. The structure of the HSPMM.

Based on analytical and numerical studies on the design parameters, the motor dimension is finalized, as shown in Fig. 2 and as tabulated in Table V. Since the supply voltage is $3 \mathrm{kV}$, the dispersion of the stator winding does not apply in high voltage. Thus form-wound windings with double layers are 
used in the HSPMM. Ventilation grooves are created in the stator slots to improve heat dissipation. Radially-magnetized NdFeB PMs are mounted on the rotor surface. The PM in each pole is divided into three segments in the circumferential direction. The gaps between poles are filled with non-magnetic non-conductive plastics to reduce the bending stress. Carbon steel is used as the rotor core and carbon fiber is employed in the retaining sleeve to improve the rotor integrity.

TABLE VII

MAIN PARAMETERS OF THE HSPMM

\begin{tabular}{|c|c|c|c|}
\hline Rated power $(\mathrm{kW})$ & 1120 & Rated speed (rpm) & 18,000 \\
\hline Rated voltage $(\mathrm{V})$ & 3000 & Rated current $(\mathrm{A})$ & 234 \\
\hline Frequency $(\mathrm{Hz})$ & 600 & Rated torque $(\mathrm{Nm})$ & 594 \\
\hline Pole number & 4 & Air gap $(\mathrm{mm})$ & 3 \\
\hline Stator slots & 27 & Stator OD $(\mathrm{mm})$ & 550 \\
\hline Stack length $(\mathrm{mm})$ & 400 & Pole arc coefficient & 0.75 \\
\hline Permanent magnet & $\mathrm{NdFeB}$ & Retaining sleeve & Carbon fiber \\
\hline
\end{tabular}

\section{NumericAl ANAlysis OF MOTOR PERFormanCE}

By using Ansys Maxwell, the performance under no load condition and full load condition are analyzed. Fig. 3 presents the induced back emf in the three-phase stator windings at no-load operation, which reads $2.91 \mathrm{kV}$. Full-load characteristics analyzed by 2D FEM are shown in Figs. 4-5. The electromagnetic torque has an average of $598 \mathrm{Nm}$, which is very close to the rated value. Fig. 5 demonstrates the phase current waveforms at full load. Its rms value is $224 \mathrm{~A}$, again, very close to the rated as well. From these simulation results, it can be seen that, the predicted performance of the motor has satisfied the specifications of the motor.

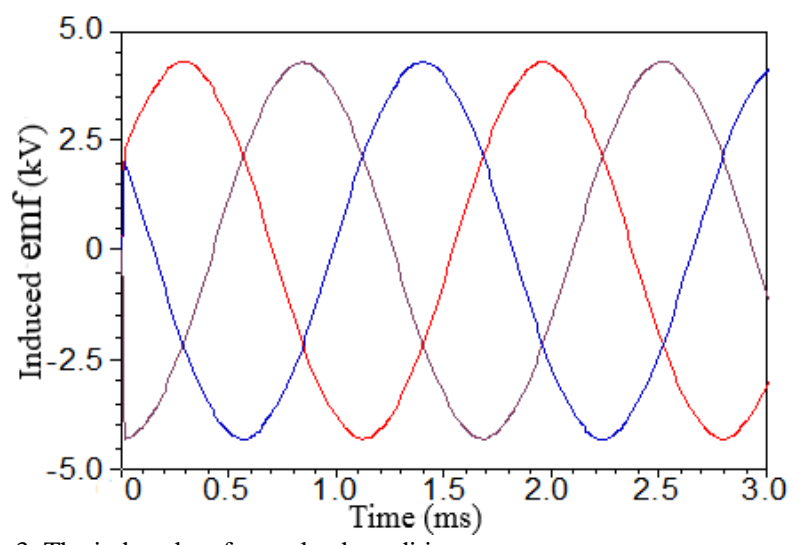

Fig. 3. The induced emf at no load condition.

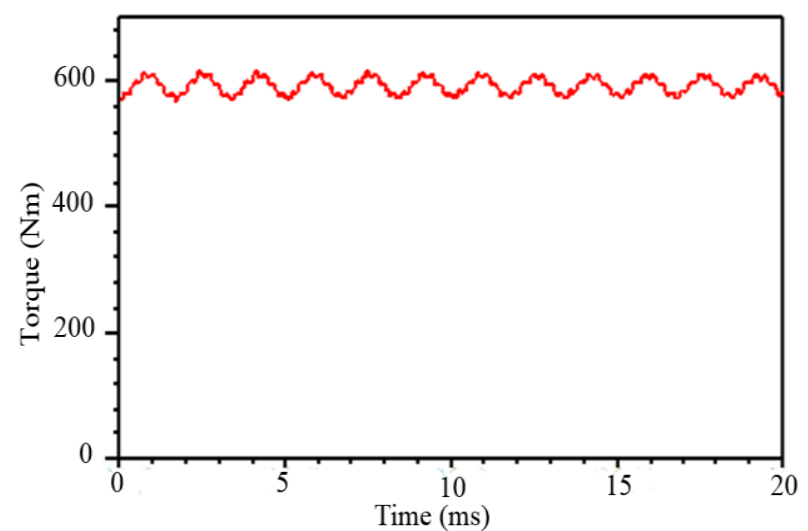

Fig. 4. The torque at full load condition.

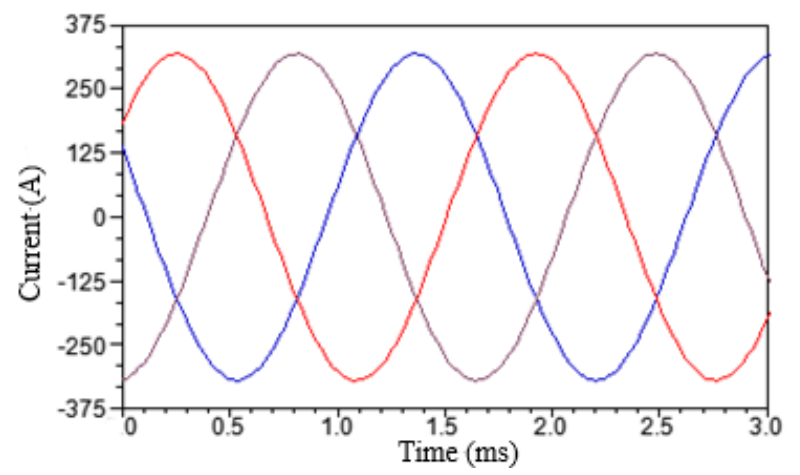

Fig. 5. The current at full load condition.

\section{LOSS AND TEMPERATURE CALCULATION OF THE HSPMM}

\section{A. Iron Loss}

During the high speed operation, the motor's core loss will be the dominant power loss in the motor. From the classic Bertotti loss separation model, the iron loss under sinusoidal flux conditions can be computed by [21]

$$
\begin{aligned}
P_{F e} & =P_{h}+P_{c}+P_{e} \\
& =K_{h} f B_{m}^{\alpha}+K_{c} f^{2} B_{m}^{2}+K_{e} f^{1.5} B_{m}^{1.5}
\end{aligned}
$$

where $P_{\mathrm{fe}}, P_{\mathrm{h}}, P_{\mathrm{c}}$ and $P_{\mathrm{e}}$ denote the core loss, hysteresis loss, classical eddy current loss, and additional eddy current loss; $K_{\mathrm{h}}$, $K_{\mathrm{c}}$ and $K_{\mathrm{e}}$ are their coefficients, respectively; $f$ is the fundamental frequency of the magnetic field; $B_{\mathrm{m}}$ is the flux density amplitude. Obviously, the rotational magnetic field is neglected in the classic model. The rotating magnetic field and alternating magnetic field simultaneously exist in the stator core.

In recent years, an orthogonal decomposition model with variable coefficients considering rotating magnetization is gaining in popularity [22]. This is presented in the following equation.

$$
\begin{aligned}
P_{\mathrm{Fe}}= & P_{\mathrm{h}}+P_{\mathrm{c}}+P_{\mathrm{e}} \\
= & K_{h} f \sum_{k=0}^{\infty} k\left(B_{\mathrm{kmax}}^{\alpha}+B_{\mathrm{kmin}}^{\alpha}\right)+K_{c} f^{2} \sum_{k=0}^{\infty} k^{2}\left(B_{\mathrm{kmax}}^{2}+B_{\mathrm{kmin}}^{2}\right) \\
& +\frac{K_{e}}{(2 \pi)^{3 / 2}} \frac{1}{T} \int_{0}^{T}\left(\left|\frac{d B_{r}(t)}{d t}\right|^{1.5}+\left|\frac{d B_{\theta}(t)}{d t}\right|^{1.5}\right) d t
\end{aligned}
$$

where $k$ represents the order of harmonics, $B_{\mathrm{r}}(\mathrm{t})$ and $B_{\theta}(\mathrm{t})$ are normal and tangential components of the stator magnetic field, respectively. $B_{\mathrm{kmaj}}$ and $B_{\mathrm{kmin}}$ represent the major axis and minor axis of $k$ order the harmonic ellipse magnetic field.

As for the iron material, silicon steel sheets (B20AT1500) with $0.2-\mathrm{mm}$ thickness are used to stack the stator core and its saturation field is greater than $1.8 \mathrm{~T}$.

The measured core loss at different frequencies with a $1-\mathrm{kg}$ core mass is shown in Fig. 6 for comparison. From Fig. 6, the core loss increases significantly with frequency. The loss coefficients $\left(K_{\mathrm{h}}, K_{\mathrm{c}}, K_{\mathrm{e}}, \alpha\right)$ obtained from the least squares method are listed in Table VIII. 


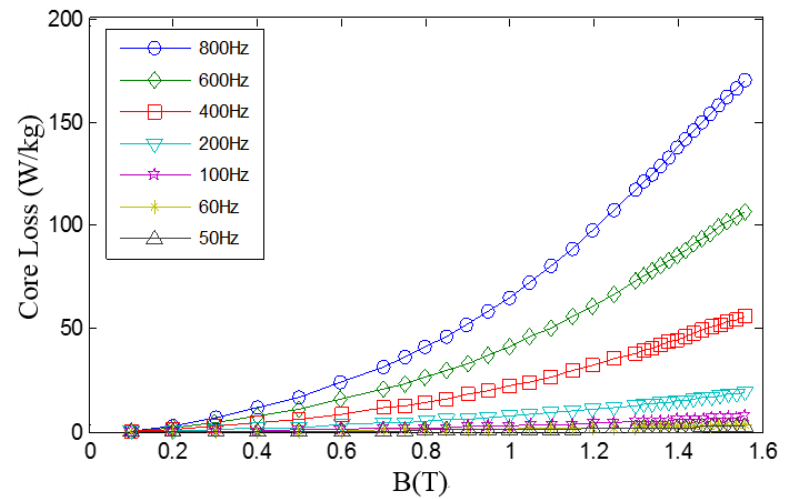

Fig. 6. Measured core loss of different frequencies.

TABLE VIII

CORE LOSS COEFFICIENTS

\begin{tabular}{|c|c|}
\hline$K_{h}$ & 0.022 \\
\hline$\alpha$ & 1.83 \\
\hline$K_{c}$ & $3.34 \times 10^{-5}$ \\
\hline$K_{e}$ & $9.55 \times 10^{-6}$ \\
\hline
\end{tabular}

The core loss is calculated via Ansys Maxwell and two analytical models based of the 2-D transient field simulation. The results are presented in Fig.7. The total core loss and classical eddy current loss based on the decomposition model are $1580 \mathrm{~W}$ and $1223 \mathrm{~W}$, respectively, both greater than these by classical Bertotti loss model. The skin effect and rotational magnetization impact on the iron loss and are needed to consider in the calculation of iron loss for high-speed motors.

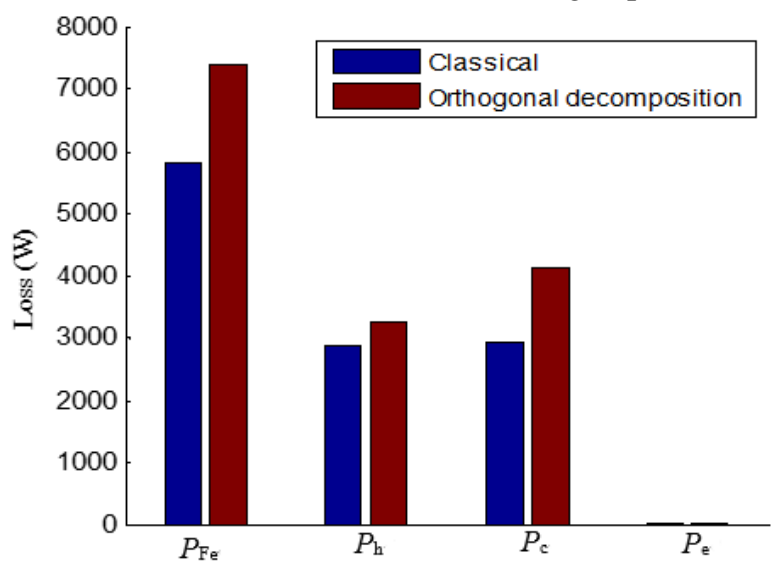

Fig. 7. Core loss results with the two different methods.

\section{B. $\quad$ Stator Winding Loss}

The total winding loss consists of conductor copper loss, and additional loss caused by skin effect and proximity effect. For the round wires, when the radius is equal to or smaller than the skin depth at the operating frequency, the $\mathrm{AC}$ resistance tends to be close to the direct current (DC) resistance [23]. However, for form-wound windings, the AC copper loss at high frequency due to the skin and proximity effects is much higher than low frequency [23]. For the developed HSPMM, the rated frequency is $600 \mathrm{~Hz}$. The high-frequency copper loss is calculated from FEM -based eddy current field simulation in Ansys Maxwell. All flat wires in form-wound windings of the stator slots are established as shown in Fig. 8. The skin effect and proximity effect become severe at high frequency, as shown in Fig. 9.

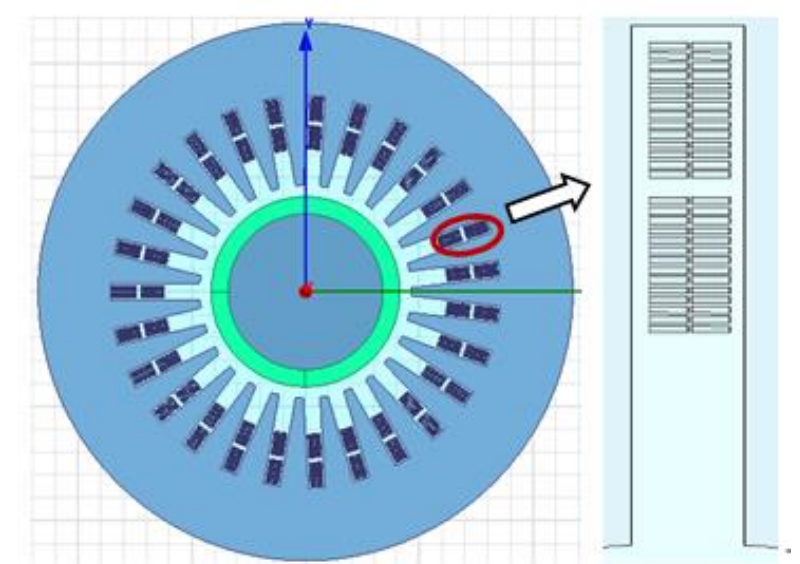

Fig. 8. Finite element model for predicting stator winding loss.

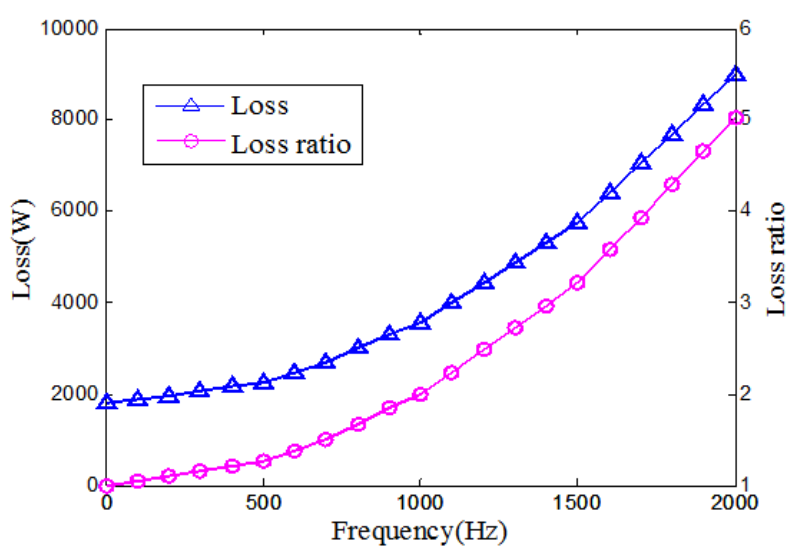

Fig. 9. Stator winding loss at different frequencies.

The winding loss as a function of frequency can be obtained by curve fitting. That is

$$
p_{a c}=0.001367 f^{2.036}+p_{d c}
$$

After removing the DC component, the addition winding loss is found by:

$$
p_{a d}=0.001367 f^{2.036}
$$

This is $3.9 \mathrm{~W}$ at $50 \mathrm{~Hz}$ and $1753 \mathrm{~W}$ for all frequency spectrum. Clearly, the additional winding loss increases significantly with frequency. The ratio of $P_{\mathrm{ac}}$ to $P_{\mathrm{dc}}$ is also plotted in Fig. 9. It can be observed that the total winding loss is about 1.3 times of the winding DC loss at $500 \mathrm{~Hz}$ and this reaches 5 times at $2 \mathrm{kHz}$.

\section{Rotor Eddy Current Loss}

The rotor eddy current loss is calculated with the 2-D time-stepping FE method in Ansys Maxwell at the rated condition. The influence of carbon fiber retaining sleeves on the rotor eddy current loss is calculated and shown in Fig. 10. The conductivity of carbon fiber is less than $5 \times 10^{4} \mathrm{~S} / \mathrm{m}$, and the conductivity of PMs is about $6.29 \times 10^{5} \mathrm{~S} / \mathrm{m}$. For low power high-speed PM motor, the influence of carbon fiber electrical conductivity on rotor total loss is very small and can be ignored. As a result, for MW high-speed PM motor, the sleeve loss is about same with PM loss when the carbon fiber conductivity is $1.5 \times 10^{4} \mathrm{~S} / \mathrm{m}$. The sleeve loss is 2 times of PM loss at $3 \times 10^{4} \mathrm{~S} / \mathrm{m}$ and it is about 3.3 times at $5 \times 10^{4} \mathrm{~S} / \mathrm{m}$. It can 
be said that conductivity of carbon fiber has a great impact on the rotor losses for MW high-speed PM motor.

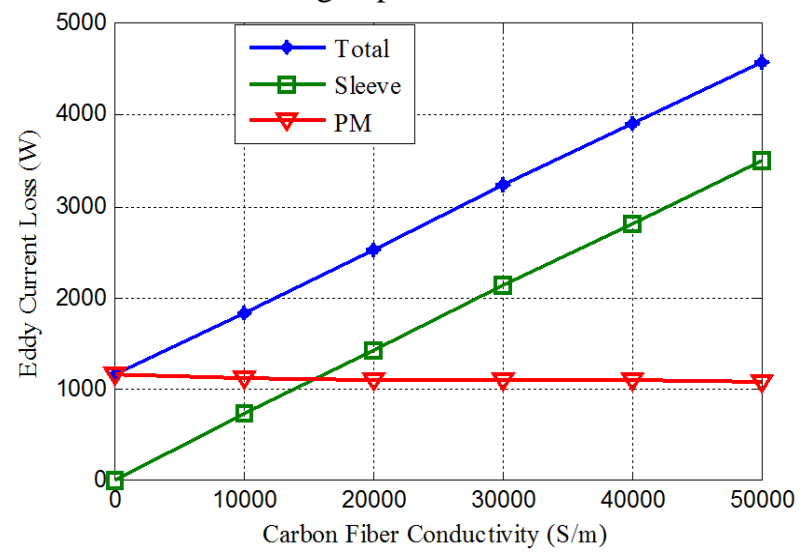

Fig. 10. Rotor eddy loss with varying carbon fiber conductivity.

\section{Air Friction Loss}

Air friction loss is usually calculated by the following empirical equation [24]

$$
p_{a i r}=K C_{\mathrm{f}} \pi \rho \omega^{3} r^{4} L_{a}
$$

where

$$
\left\{\begin{array}{l}
C_{\mathrm{f}}=\frac{0.0152}{\mathrm{R}_{\mathrm{e} \delta}}\left[1+\left(\frac{32 \mathrm{R}_{\mathrm{e} a}}{7 \mathrm{R}_{\mathrm{e} \delta}}\right)^{2}\right]^{0.38} \\
\mathrm{R}_{\mathrm{e} \delta}=\frac{\rho \omega r \delta}{\mu} \\
\mathrm{R}_{\mathrm{ea}}=\frac{\rho v_{\mathrm{a}} 2 \delta}{\mu}
\end{array}\right.
$$

where $\rho$ is the air density, $\omega$ is the angular velocity, $L_{a}$ is the stack length and $r$ is the rotor radius, $C_{f}$ is the friction coefficient of air, $R_{\mathrm{e} \delta}$ and $R_{e a}$ are the tangential Reynolds number and the axial Reynolds number, respectively, $\delta$ is the air-gap length, $v_{\mathrm{a}}$ is the average axial velocity and $\mu$ is the air's dynamic viscosity.

Alternatively, an FEM-based fluid field in Ansys CFX is developed to calculate the air friction loss [24]. The losses calculated by analytical and FEM methods are presented in Fig. 11.

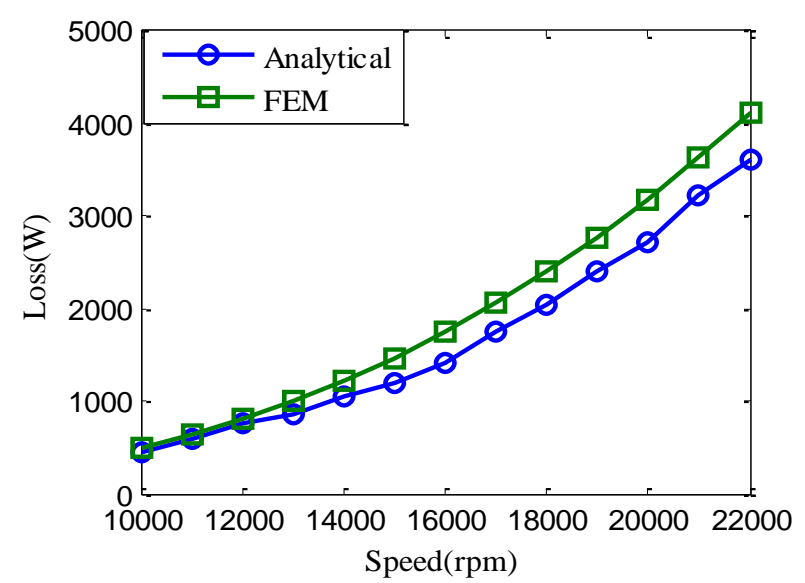

Fig. 11. The predicted air friction losses by the two different methods.
The predicted losses from the FEM are generally greater than the analytical ones, especially at high speeds. The relationship between the loss and speed can be obtained by curve fitting. That is

$$
p_{\text {air }}=k_{1} n^{2.69}
$$

where, $k_{1}$ is the air friction coefficient, $n$ is the speed.

\section{E. Temperature Analysis of HSPMM}

In order to remove the generated heat in the rotor, a cooling system is designed to utlize both air and water cooling. The cooling air path is set in the stator slot, and a cooling water loop in the stator housing. The machine temperatures under rated load are calculated using the fluid-solid coupling method in Ansys CFX. The machine temperature distributions have been shown in reference [25]. The maximum temperatures of the PM rotor is about $145^{\circ} \mathrm{C}$ and it is much lower than the critical temeprature of PM (about $180^{\circ} \mathrm{C}$ ). The temperatures of the windings and stator core are about $76^{\circ} \mathrm{C}$ and $70^{\circ} \mathrm{C}$. The temperature of the end-winding (about $69^{\circ} \mathrm{C}$ ) is lower than the middle of the winding because of better ventilation at the location.

\section{EXPERIMENTAL TESTS AND RESULT ANALYSIS}

Based on the electromagnetic design, the HSPMM is prototyped and experimental tested. The stator, rotor and shell are shown in Fig. 12 and experimental test facilities are shown in Fig. 13 [26]. In Fig. 13(a), no-load motor tests are carried out to verify the motor design. Experimental loads test is carried out, as shown in Fig. 13(b). Test results are presented in Figs. 14-16, Table IX.

No-load emf voltage is an important indicator for motor tests. In order to get the performance at different speeds, the HSPMM is driven by an induction motor through a gearbox. Fig. 14 presents the induced line voltage at different speeds by FEM and measured results for comparison. At no-load condition, the open-circuit induced line voltage at rated speed measures 2.91 $\mathrm{kV}$, which is nearly the same as the designed rated voltage. Clearly, a good agreement can be found between prediction and measurements in Fig. 14.

The no-load phase currents against speeds $(3,000-18,000$ rpm) running as a motor are measured by Fig. 13(a). The measured no-load current against speed is plotted in Fig. 15. It is shown that the no-load phase current is $21 \mathrm{~A}$ at the rated speed, which reasonably agrees with the simulation (17 A).

At load tests, the HSPMM drives a MW induction machine through a gearbox. The prototype and load (an induction machine) are controlled by two inverters. The speed of the prototype and load machine can be regulated by the PWM frequency of the inverter and gearbox, respectively. Fig. 16 presents current waveform at full-load and 18,000 rpm. The winding temperatures are measured by three thermistors inserted in the winding ends. Measured and calculated results for the full load at the speed of 18,000rpm are shown in Table IX. At 18,000 rpm, the phase current, line voltage and winding temperature measure $235 \mathrm{~A}, 3140 \mathrm{~V}$ and $65^{\circ} \mathrm{C}$. The numerical and experimental results are also listed in Table IX for comparison and all results agree well with numerical 
calculations. The results show that the prototype motor has excellent performance at full load.

Overall, it can be said that the proposed motor design is proven to be effective and developed numerical models are reasonably accurate to predict the motor's electromagnetic performance.

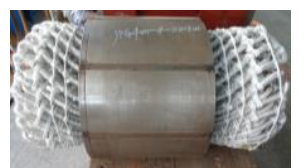

(a)

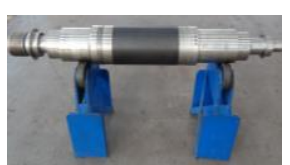

(b)

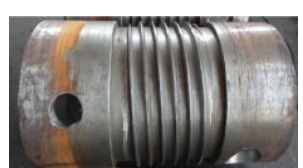

(c)
Fig. 12. The prototype motor. (a) Stator. (b) Rotor. (c) Shell.

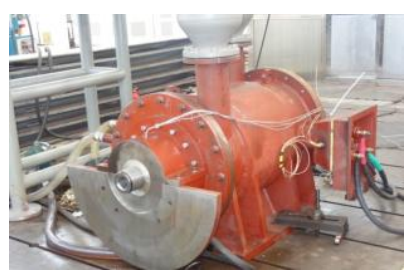

(a)

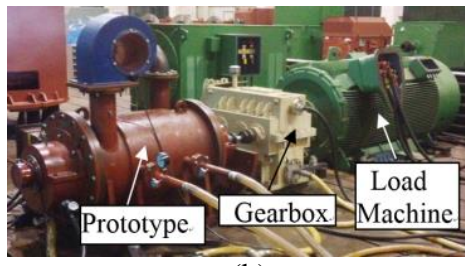

(b)
Fig. 13. Experimental test facilities. (a) Experimental test rig for no-load motor conditions. (b) Experimental test rig at load motor conditions.

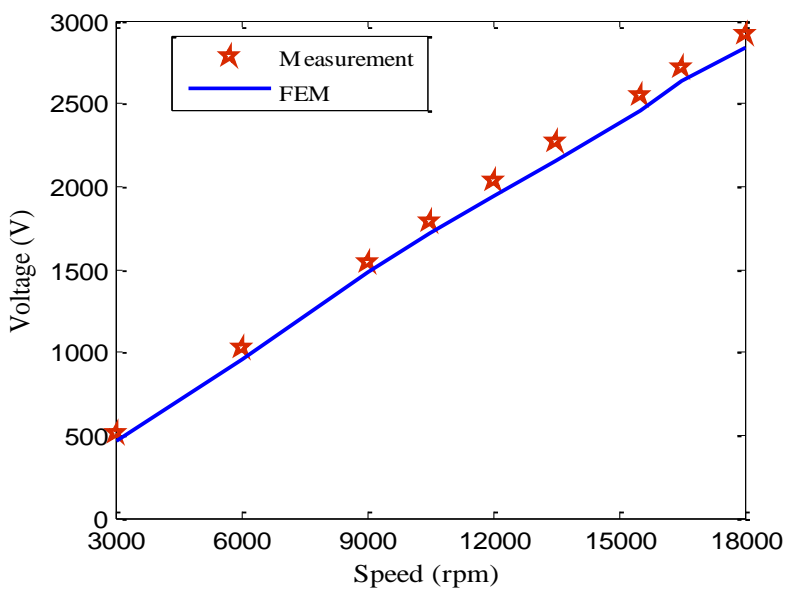

Fig. 14. The no-load induced line voltage against speed.

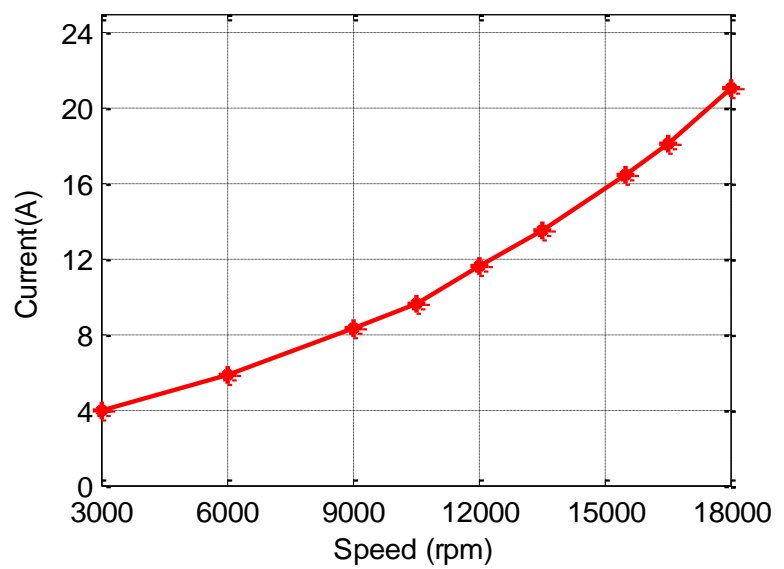

Fig. 15. The measured no-load current against speed at run as a motor.

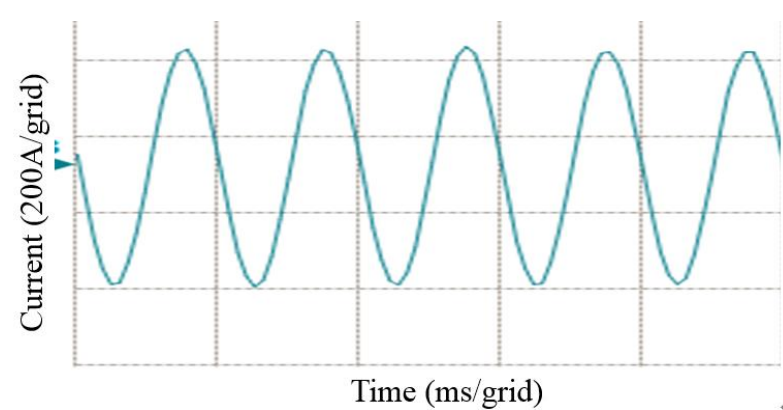

Fig. 16. Waveform of the full-load current at rated speed.

TABLE IX

RESUlTS For FULl LOAD AT RATED SPEED

\begin{tabular}{|c|c|c|c|c|c|}
\hline Item & $\begin{array}{c}\text { Input } \\
\text { power } \\
(\mathrm{kW})\end{array}$ & $\begin{array}{c}\text { Line } \\
\text { voltage } \\
(\mathrm{V})\end{array}$ & $\begin{array}{c}\text { Phase } \\
\text { current } \\
(\mathrm{A})\end{array}$ & $\begin{array}{c}\text { Power } \\
\text { factor }\end{array}$ & $\begin{array}{c}\text { Winding } \\
\text { Temperature } \\
\left({ }^{\circ} \mathrm{C}\right)\end{array}$ \\
\hline Measurement & 1150 & 3140 & 235 & 0.965 & 69 \\
\hline Calculation & 1147 & 3000 & 224 & 0.96 & 65 \\
\hline
\end{tabular}

\section{CONCLUSION}

This paper has described the design of a high-speed permanent magnet motor for compressor applications. The study on power losses is carried out by analytical, numerical and experimental approaches. The motor parameters including pole number, stator slot number, rotor dimension, air gap length, PM material, thickness and pole arc are optimized. The no-load and full-load performance is investigated by the $2 \mathrm{D}$ FEM and experiments. The total loss in the HSPMM (including iron loss, stator winding loss, rotor eddy current loss and air friction loss) have been predicted and measured. Test results from simulation and experiments validate the effectiveness of the proposed models and prototyped motor. The developed techniques are highly relevant to high-power high-speed applications such as compressors, pumps, ships and traction drives.

\section{REFERENCES}

[1] F. X. Wang, "Study on design feature and related technology of high speed electrical machines," Journal of Shenyang University of Technology, Vol. 28, No. 3, pp. 258-263, Jun. 2006.

[2] J. J. H. Paulides, G. W. Jewell, D. Howe, "An evaluation of alternative stator lamination materials for a high-speed, $1.5 \mathrm{MW}$ permanent-magnet generator," IEEE Trans. Magn., Vol. 40, No. 4, pp. 2041-2043, Jul. 2004.

[3] H. B. Qiu, R. Yi, W. L. Li, N. Jin, "Influence of rectifiers on high-speed permanent magnet generator electromagnetic and temperature fields in distributed power generation systems," IEEE Trans. Energy Conversion, Vol. 30, Issue: 2, pp. 655-662, Jun. 2015.

[4] D. K. Hong, B. C. Woo, Y. H. Jeong, D. H. Koo, C. W. Ahn, "Development of an ultra high speed permanent magnet synchronous motor," International Journal of precision engineering and manufacturing, Vol. 14, No. 3, pp. 493-499, MAR. 2013.

[5] M. Pinilla, "Performance improvement in a renewable energy direct drive permanent Magnet machine by means of soft magnetic composite interpoles," IEEE Trans. Energy Conversion, Vol. 27, Issue: 2, pp. 440-448, Jun. 2012.

[6] Z. Q. Zhu, K. Ng, and D. Howe, "Design and analysis of high-speed brushless permanent magnet," IEE Electric Machines and Drives Conference (EMD'97), Sept. 1-3, Conf. Pub. 444, pp.381-385, 1997.

[7] D. Gerada, A. Mebarki, N. L. Brown, C. Gerada, A. Cavagnino, A. Boglietti, "High-speed electrical machines: technologies, trends, and developments," IEEE Trans. Ind. Elec., vol. 61, no. 6, pp. 2946-2959, Jun. 2014 
[8] D. Borkowski, T. Wegiel, "Small hydropower plant with integrated turbine-generators working at variable speed," IEEE Trans. Energy Conversion, Vol. 28, Issue: 2, pp. 452-459, Jun. 2013.

[9] M. A. Rahman, A. Chiba, and T. Fukao, "Super high speed electrical machines: Summary," in Proc. IEEE Power Eng. Soc. (PES) Meet, Jun. 6-10, 2004, vol. 2, pp. 1272-1275.

[10] Z. Kolondzovski A. Arkkio J. Larjola J, P. Sallinen, "Power limits of high-speed permanent-magnet electrical machines for compressor applications," IEEE Trans. Energy Conversion, vol. 26, no. 1, pp. 73-82, Mar. 2011.

[11] N. Bianchi, S. Bolognani, and F. Luise, "Potentials and limits of high-speed PM motors," IEEE Trans. Ind. Appl., vol. 40, no. 6, pp. 1570-1578, Nov./Dec. 2004.

[12] J. N. Dong, Y. K. Huang, L. Jin, H. Y. Lin, H. Yang, "Thermal optimization of a high-speed permanent magnet motor," IEEE Trans. Magn., vol.50, no. 2, pp. 749-752, Feb. 2014.

[13] A. Tenconi, S. Vaschetto, A. Vigliani, "Electrical machines for high-speed applications: design considerations and tradeoffs," IEEE Trans. Ind. Elec., vol. 61, no. 6, pp. 3022-3029, Jun. 2014.

[14] A. Castagnini, M. Garavaglia, F. Moriconi, and G. Secondo, "Development of a very high speed and power synchronous PM motor," Int. Conf. Electrical Machines (ICEM'02), Brugges, Belgium, Aug. $25-28,2002$.

[15] J. Luomi, C. Zwyssig, A. Looser, and J.W.Kolar, "Efficiency optimization of a 100-W 500000-r/min permanent-magnet machine including airfriction looses," IEEE Trans. Ind. Appl., vol. 45, no. 4, pp. 1368-1377, Jul./Aug. 2009.

[16] W. L. Li, H. B. Qiu, X. C. Zhang, J. C. Cao, S.N. Zhang, R. Yi, "Influence of rotor-sleeve electromagnetic characteristics on high-speed permanent-magnet generator," IEEE Trans. Ind. Elec., vol. 61, no. 6, pp. 3030-3037, Jun. 2014

[17] T. Y. Wang, "Study on combined design and dynamic characteristics of rotor for high-speed PM machine," Ph.D. dissertation, Shenyang University of Technology, Shenyang, China, 2010.

[18] N. Huang, "Design and loss analysis of MW-level high speed permanent magnet synchronous machine," M.S. dissertation, Shenyang University of Technology, Shenyang, China, 2013.

[19] S. K. Chen, Machines design. Beijing, China: Machinery Industry Publishing, 2000

[20] X. H. Wang. Permanent Magnet Machines. Beijing, China: China Electric Power Publishing, 2011.

[21] G. Bertotti, "General properties of power losses in soft ferromagnetic materials," IEEE Trans. Magn., vol. 24, no. 1, pp. 621-630, Jan. 1988.

[22] X. G. Kong, F. X. Wang, Y. Xu, "Analysis and calculation of iron losses of high-speed permanent magnet machines," Electric machines and control, Vol. 14, No. 9, pp. 26-30, Sep. 2010.

[23] D. A. Gonzalez, D. M. Saban, "Study of the copper losses in a high-speed permanent-magnet machine with form-wound windings," IEEE Trans. Ind. Elec., vol. 61, no. 6, pp. 3038-3045, Jun. 2014

[24] J. Xing, F. Wang, D. Zhang, "Research on rotor air friction loss of high-speed permanent magnet machines", Proceedings of the CSEE, Vol. 27, No. 30, pp. 14-19, Oct. 2010.

[25] F. Zhang, G. Du, T. Wang, F. Wang, W. Cao, D. Wang, "Integrated design of 1.12MW high speed PM machine based on multi-physics fields," Transactions of China Electrotechnical Society, vol. 30, no. 12, pp. 171-180, Jul. 2015

[26] F. G. Zhang, G. H. Du, T. Y. Wang, G. W. Liu, W. P. Cao, "Rotor retaining sleeve design for a 1.12-MW high-speed PM machine," IEEE Trans. Ind. Appl., vol. 51, no. 5, pp. 3675-3685, Sept./ Oct. 2015.

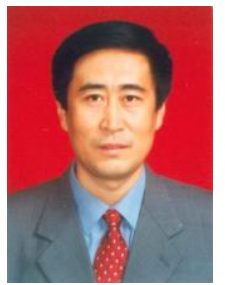

Fengge Zhang was born in 1963. He received the B.E.E., M.S., and Ph.D. degrees from Shenyang University of Technology, Shenyang, China, in 1990, and 2000, respectively, all in electrical engineering. Since 1984, he has been serving as a Teacher with the School of Electrical Engineering, Shenyang University of Technology, where he is currently a Professor. He has been confirmed as young academic skeleton by Liaoning Province and the National Machinery Industry Ministry, respectively. From October 2001 to July 2002, he was a Visiting Scholar with Esslingen University of Applied Sciences, Esslingen am Neckar, Germany. His research and teaching interests include electric-magnetic theory, dynamic simulation, magnetic field analysis, optimized design, computer control technology of electrical machines, and wind power generating systems. He is also active in the area of power converters for variable-speed control and drive systems.

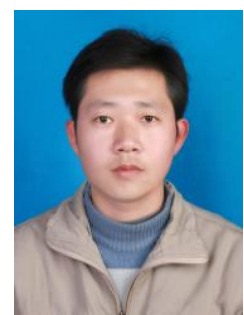

Guanghui Du was born in Henan, China, in 1987. He received his B.S. degree in Electrical Engineering from the Qingdao Agricultural University, China, in 2010. He worked toward his M.S. degree from Shenyang University of Technology from 2010 to 2012. He presently studies as a Ph.D. student at the Institute of Electric Control Technology, Shenyang University of Technology. His current research interests include design and calculation of a high-speed motor.

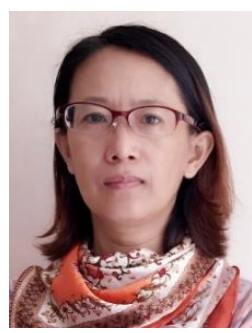

Tianyu Wang received the B.Eng in mechanical engineering from Liaoning Technical University, Liaoning, China, in 1991, and the Ph.D. degree in electrical machines and drives from Shenyang University of technology, in 2010. She is currently a professor in Shenyang Institute of Engineering. Her research interests are in high speed machine and mechanical strength and multi-physical fields coupled analysis.

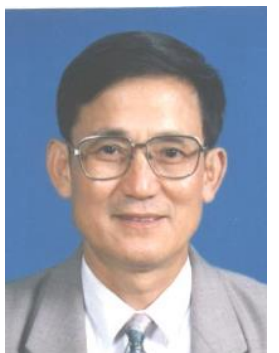

Fengxiang Wang was born in Shandong Province, China, in 1938. He received the B.E.E. and M.S. degrees from the Tsinghua University, Beijing, China, in 1962 and 1966 respectively. In 1966, he joined the Shenyang University of Technology, Shenyang, China, where he is currently a Professor. From 1981 to 1983, he was a Visiting Scholar at the University of Wisconsin, Madison, US. From 1992 to 1993, he was a Senior Visiting Scholar at the University of Toronto, Toronto, Canada. As a Visiting Professor from 1996 to 1997, he worked in the Department of Electrical Engineering, the Ohio State University, Columbus, US. He had published more than 90 academic papers. His major interests are electrical machines and drives, power electronics, and electromagnetic field analysis.

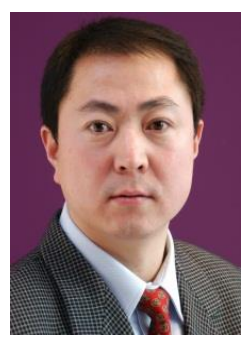

Wenping Cao (M'05-SM'11) received the B.Eng in electrical engineering from Beijing Jiaotong University, Beijing, China, in 1991, and the Ph.D. degree in electrical machines and drives from the University of Nottingham, Nottingham, U.K., in 2004. He is currently a Marie Curie Fellow with the Department of Electrical Engineering and Computer Science, Massachusetts Institute of Technology, Cambridge, MA, U.S.A. and a Chair Professor of Electrical Power Engineering with Aston University, Birmingham, U.K. His research interests include fault analysis and condition monitoring of electric machines and power electronics.

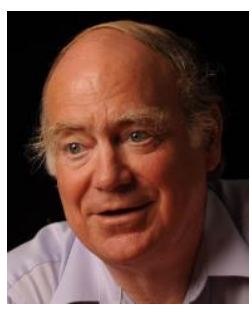

James L. Kirtley (LF'91) received the Ph.D. degree from the Massachusetts Institute of Technology (MIT), Cambridge, MA, USA, in 1971. He is a Professor of Electrical Engineering with the Department of Electrical Engineering and Computer Science, School of Engineering, MIT. He was with the Department of Large Steam Turbine Generators, General Electric as an Electrical Engineer, and with Satcon Technology Corporation as the Vice President and the General Manager of the Tech Center, and as a Chief Scientist and a Director. He was Gastdozent with the Swiss Federal Institute of Technology, Zurich, Switzerland. His research interests include electric machinery and electric power systems.

Prof. Kirtley served as the Editor-in-Chief of the IEEE TRANSACTIONS ON ENERGY CONVERSION from 1998 to 2006 and continues to serve as an Editor for the journal. He was the recipient of the IEEE Third Millennium Medal in 2000 and the Nikola Tesla Prize in 2002. He was elected to the U.S. National Academy of Engineering in 2007 